\title{
SURVEY ON CACHING AND REPLICATION ALGORITHM FOR CONTENT DISTRIBUTION IN PEER TO PEER NETWORKS
}

\author{
C.SriguruLakshmi ${ }^{1}$, G.sivakumar ${ }^{2}$, V.Venkatachalam ${ }^{3}$ \\ ${ }^{1}$ PG Scholar, Dept of CSE, Erode Sengunthar Engg College, India \\ ${ }^{2}$ Assistant Professor-SG, Dept of CSE, Erode Sengunthar Engg College, India \\ ${ }^{3}$ Principal, Dept of CSE, The Kavery Engg College, India
}

\begin{abstract}
In this paper, we focuses on caching and replication algorithm for content distribution in peer to peer networks. Caching and replication is a key strategy for improve the reliability, availability, and performance in peer to peer networks. This paper gives a brief introduction to caching, replication and various algorithms have been discussed and a detailed study has been performed. The comparison table shows it clearly that an algorithm satisfies the caching and replication requirement.
\end{abstract}

\section{Key words}

Caching, Replication, P2P networks, Comparison of algorithm

\section{INTRODUCTION}

P2P systems are classified into two types namely: 1). Centralized P2P system and 2). Decentralized P2P system. The paper mainly deals with decentralized P2P system. Decentralized $\mathrm{P} 2 \mathrm{P}$ system can be classified into decentralized structured and unstructured system. In Decentralized Structured P2P architecture network topology is tightly controlled, whereas Decentralized Unstructured P2P systems do not have any control over the network topology, and placement files over the network. The most general $\mathrm{P} 2 \mathrm{P}$ system is the decentralized unstructured system .Peers form a network among them on top of the existing inter infrastructure, which is known as the Overlay Network. The challenges facing in P2P systems are scalability, reliability, access latency, network traffic, fault tolerance, bandwidth utilization, security, and load balancing. Data replication and caching techniques are the important two services in peer to peer networks. It increases data availability by creating local or nearly available copies of popularly used items, by forwarding each query to its nearest copy; the query search latency can be effectively reduced. It also reduces communication overhead, increased system performance, achieves fault-tolerance, and enhances reliability and load balancing.[17]. 1)Reliability: In p2p results in the elimination of the single point of failure that has dogged all timesharing systems. It also supports replication for all of its network services. If one of the servers becomes unavailable, a client automatically switches over to one of the replicated servers. 2)Availability: The wining

DOI : $10.5121 /$ ijcseit.2013.3503 
feature of content distribution components are available to users at all times. Use of replication enables an administrator to do file system backups while the system is up and running. The replicated copy remains stable even while the user is changing the original file. 3)Fault Transparency or Tolerance: Components in a distributed system can fail independently. A file may be made more available in the face of failures of a file server if it appears on more than one file server. Availability is one of the main measures of the advantage of a replication algorithm. 4)Load Balancing : Replication of files in a p2p networks allows better load balancing. 5)Performance: Another advantage of a $\mathrm{p} 2 \mathrm{p}$ system is the ability to share information with many diverse users. DFS is an efficient, extensible system. In addition, the server tracks which clients have cached copies of files, reducing the need for the client to constantly query the server, as well as reducing the network and server load.

\section{CACHING TECHNIQUE}

Caching is processed on the media chunks. The caching is done in media chunks in two ways. The first way is to cache the chunks in a predefined manner when no data regarding the popularity of the media is available. The second way caching is work working based on the popularity of the video. For example, when a server receives a request from a client, it immediately responds to the client if the object is in its local cache storage space. In cache storage space storing frequently used videos. Cache using various algorithms to store the video contents. So Caching increase the searching speed, hit rate and reduces the client waiting time and communication among peers.

\section{REPLICATION TECHNIQUES}

Replication is the process of creation and maintenance of duplicate copies of objects in internetscale distributed system. Replication improves the system performance, fault tolerance, reduces a network bandwidth usage and increases the availability of popular data objects by distributing the source of information in globally. To efficiently use the server storage we need to replicate objects that will yield the best performance. Replication is needed in the case of System failure, network traffic and to increase system scalability, load balancing, and to reduce access cost. For example, users can access a local object rather than origin server to minimize network traffic, access latency and provide location transparency.[17] There are different models of object replication. We mainly deal with a distributed replication group. A distributed replication group contains several servers dedicating some storage for the replicas. A server has to serve requests from its clients and also from other servers in the group. When a server receives a request from a client, it immediately responds to the client if the object is in its local storage. Otherwise, the object is fetched from other servers within the group at a higher access cost or from the origin server, at an even higher cost; in the case no server within the group stores a replica of the object. The purpose of the replication group is to achieve minimum access cost.

\section{RESEARCH ISSUES IN CACHING AND REPLICATION TECHNIQUES}

In this paper we have analyzed only some methods of caching and replication algorithms in $p 2 p$ networks as follows: 
International Journal of Computer Science, Engineering and Information Technology (IJCSEIT), Vol.3,No.5,October 2013

\section{A. Cache Optimization On Hot-Point Proxy Caching Using Weighted-Rank Cache Replacement Policy(WRCP)}

Existing Approach the HP-Proxy model using Dual Cache Replacement Policy(DCRP). DCRP function, only calculate the access frequency percentage and other costs to calculate the weighted cost(WC). Based on the WC, the three-level cache replacement is performed, and even the parts of the objects that have not been accessed recently or are very old are kept. The DCRP does not consider the age or access gap of the objects currently available in the proxy server.In Proxy Caching schemes to provide better media content delivery. It store the initial part or interleaved parts of video objects into a proxy server's cache. If the cache is done, first access by a client from the media server. Not possible to cache an entire object in proxy. The media objects need to divided into smaller chunks. The cache is processed on the media chunks.[3]

Weighted-Rank Cache Replacement Policy(WRCP):

- Access Frequency of object - no.of times accessed compared with all available objects.

- Mean age of the object - mean age of object compared with all available objects.

- Mean access Gap ratio of the object - it calculate at every mean arrival time.

- Other cost functions- It says fetching latency and size of the object.

Weighted Rank Calculation (WR): The WR is used to determine object to the users. The objects with a high ranking are kept in the cache without replacement. Each object with a medium or low ranking is selected as a victim object and replaced with either an entire object or a partial object from the cache. The WR of an object is assigned based on the calculated WC value of the object.

\section{B. Design and Analysis of Streaming For P2P VOD with MDC}

P2P video streaming system reduced server load compared to traditional client server system. More and more video on demand system using $\mathrm{p} 2 \mathrm{p}$ service. The user requirement is satisfied by another user. So it reduced the server loading. Existing System using Cache and Relay Scheme. Each peer cache the most recent video segment in its buffer and then relay on late joining peers. This scheme depends on buffer size. User require service to the server, it increase the server load.MDC: (Multiple Description Coding). It uses MDC. It divided the video file into segments, divided those segments again by using MDC that encoded the several description. It solve the package losing problem, and allowed users with different bandwidth can all watch the video.It satisfying user's QOS(Quality Of Service). Even if the pocket loss can still view the lower video quality. And it reduced high churn rate.[1]Video locality Based Buffering: video divided into segments. Classified each segments into two classes. Leading, non leading. Leading segment store the popular videos. And non leading segment store some consecutive videos. When the buffer is full, it implements LRU(least recently used) mechanism that release non popular video segments. Balanced Dynamic Buffering: It using inter group balancing and intra group balancing. Intra group balancing determines with the least aggregate bandwidth. Inter group having most aggregate bandwidth. When the peer buffer is full, it drops the cached description with most required bandwidth. Dynamic buffering achieves a more efficient utilization of peer resources. Streaming Mechanism: MDC provides the complete process. It defines i) from peer joining the system to leaving after viewing the whole video, ii)after peer joining the system, 
peers are grouped by server to received leading description number. Caching Mechanism: It caches the frequently watching videos. It gets leading segment description of group from peer zof other group until quality of the segment is satisfied to continue caching the next segment. When the buffer is full, buffer needs to be adjusted by buffering mechanism. Buffering Mechanism: This mechanism using non-leading, secondary, leading segment. When the buffer is full, the first released non leading buffer. It using FIFO mechanism to released non-leading segment. But buffer is still full and can't receive the subsequent segment description. After it released secondary segment. It using MRU(Most Recently Used) mechanism to released secondary segment.

\section{Design of Distributed Prefetching Protocol in Push to Peer Video on Demand $\operatorname{System}(\mathrm{FSS} \&$ CBS)}

In $p 2 p$ streaming system, the upstream bandwidth of peers are larger than video playback rate, this $\mathrm{p} 2 \mathrm{p}$ system does not overcome the upstream bandwidth limitation. In Existing proxy prefix caching technique require upstream bandwidth of peer larger than video play back rate. So it goes for push to peer system. In push to peer system does not relay on content server. So this system can overcome the bandwidth limitation. It using content placement and associated pull policies. In content placement increase the content availability and improve the client bandwidth utilization. The mostly wanted videos are proactively fetched to the set of boxes in the subscriber during time of low network utilization(eg: Early morning). The client can easily download and playing the videos in set top box. So it reduced server load and client waiting time.It using 2 approaches: Full Striping scheme, Code based placement scheme.[2] Push-to-Peer System: It willing to have content proactively push to them before video distribution among the cooperating peers begins.Full Striping Scheme: Videos are strip into video blocks and push the different video block into the set top box. This system provide high quality video streaming and it reduce the client waiting time, network congestion. In this scheme stripes each window of overall movie $\mathrm{M}$ boxes. Every window divided M blocks. Each of size is W/M. Each block pushed to only one box. Each box stored different blocks of window. Code Based Placement Scheme: Videos are encoded into coded symbol, by using the rate less code algorithm. This algorithm eliminate the box of failure in full striping scheme. Rate less code algorithm generate an infinite number of coded symbol. It fixes the rate of each video content. To assign the symbol of each video content. This system easy to find the most probable video. Distributed Prefetching Protocol: When the client's required video not available in the set top box, then the distributed prefetching protocol used to directly connect client to the video server and streaming the video server to client.

\section{Popularity Aware Limited Caching for Reliable on Demand P2P video Streaming(PALC)}

The peer to peer network as a good environment for resource sharing over the Internet. Compared with traditional file sharing provokes a significant amount of today internet traffic . It has insufficient memory, high bandwidth utilization for large scale media objects, and lack of cooperation between proxies and their clients. Very challenging task to achieve efficient content delivery under the increased availability of continues media streaming.[5]Existing System: Proxy based caching, Segment Based-proxy caching Proposed System: Hybrid Proxy caching Proxy Based Caching: The proxy caching provides significant performance improvement in video streaming with asynchronous demands and highly localized access interests. proxy caching that can achieve efficient streaming for small size video content. In large video content, caching a 
complete media objects is not practically feasible in the case of limited proxy cache space. Segment-Based Proxy Caching: To handle the large size media objects. It caching the entire media content, the segment based approach partially caches the segments of media objects. The segment-based proxy caching technique provides efficient streaming with high scalability. Reason's)The small size of the reference locality of video objects leads to a cache space problem in media proxy systems.ii)The single point failure may occur in proxy based caching system.iii) It is highly vulnerable to overload.iv) Reservation of proxy bandwidth for continuous streaming.Hybrid Proxy Caching: An efficient hybrid proxy caching system in order to reduce the network/ server load. It improving the end user video quality. It also an efficient Popularity Aware Limited Caching (PALC) algorithm that cooperatively utilizes the memory space of the proxy servers and clients to achieve efficient and cost effective P2P video streaming. The proxy maintains the popular media segments for global sharing. In order to maintain the unpopular segments, a small space is left for each peer in the network. The overall hit ratio is improved due to the effective and adaptive maintenance of popular and unpopular segments. It uses as follows.

- Video Segmentation

- Distributed hash table(DHT)

- Announcing of video media segments

- Cache Lookup Retrieval Algorithm

\section{E. On Replication Algorithm in P2P VoD(RLB \& ARLB)}

In $\mathrm{p} 2 \mathrm{p} \mathrm{VoD}$ contributes some storage to store the videos. Peers have the sufficient bandwidth for given playback rate.[4] The relationship between the storage capacity at each peer? This storage capacity based on the number of videos, number of peers, and off-loading of video server bandwidth. We use simple statistical model to drive the relationship. It using generic replication algorithm random with load balancing. It balances the both Homogenous and Heterogeneous peers. Proposed System: Replication algorithm with Random load balancingExisting System: RLB-Centralized algorithm.RLB-Centralized Algorithm: It is expansive to use a centralized algorithm, RLB to push movies to peers for storage, It implement Reactive and Distributed replication algorithm, Replication at each peer is changed only after the peer finished viewing a movie. Replication decision whether stores the movie locally or discard it. Reactive algorithm is adapting the system dynamics. (Eg: movie popularity churn, and peer churn).The centralized algorithm like RLB is static and not very practical in system dynamics.Replication Algorithm:(RLB): It produce best load balance among the peers, terms of average load and correlation of load. We use a generic algorithm called Random with Load Balancing. It balances the average load and reduced the different correlation load. It efficient to utilize the bandwidth. It provides the scaling properties of what $\mathrm{p} 2 \mathrm{p}$ replication can achieve. It servers the thumb rule: needed peers storage for given number of movies. These peers are Homogenous and Heterogeneous. It using RLB Distributed and Adaptive Algorithm(ARLB) and how to coverage to a balanced state given movie popularity. Random Demand Model: Random algorithm only generates the replication allocation. That balances the load for all movies.Adaptive RLB: Movie replication viewed movies only. After a peer i viewed a movie $\mathrm{j}$, the peer runs the Adaptive RLB replication algorithm. It achieves balanced bandwidth for all movies. And it easily achieved by centralized algorithm in RLB.It achieves the adaptation. 


\section{F. Exploring the Optimal Replication Strategy in P2P-VoD Systems: Characterization and Evaluation(ORA)}

We address an important open problem: what is the "optimal replication ratio" in a $\mathrm{P} 2 \mathrm{P}-\mathrm{VoD}$ system such that peers will receive service from each other and at the same time, reduce the access to the content server? Issues: 1) what is the optimal replication ratio of a movie if we know its popularity, and 2) how to achieve these optimal ratios in a distributed and dynamic fashion. In Existing approach using the proportional replication strategy is "suboptimal". In Proposed approach expands the design space to both "passive replacement policy" and "active push policy" to achieve the optimal replication ratios. It shows how to greatly reduce server's workload and improve streaming quality via our distributed algorithms. Two types of Deployment: 1.software based and 2.set-top box based. 1.Software Based- Active peers 2.Set top box Based- Non active peers[7]Peer Scheduling Policy: A peer first seeks help from concurrent peers; if the playback rate cannot be satisfied, the peer seeks help from replication peers; if the playback rate still cannot be satisfied, the server $S$ will upload the data to this peer so as to satisfy the playback rate.Suboptimality of the Proportional Replication Strategy:This replicate more unpopular movies. Optimal ratio of the Optimal Replication in set top box system:It only replicates popular movies. It achieves optimal ratio.

\section{G. Home-Box-assisted Content Distribution Network for Internet Video-on-Demand Services(HB)}

The paper an adaptive popularity-based video caching strategy among the introduced HB layer and compares it through simulation to existing CDNs' one. In Existing approach CDNs, the video contents are pushed from a Content server to multiple powerful servers.[6] so called surrogates. In replicas placement, content clustering, client request redirection consider pure CDN. It consumes high storage and uploads connectivity. In proposed approach introduces a new equipment in the Home-Box virtual layer, capable of content caching and with streaming capabilities, permitting through the deployment of an overlay virtual layer to overcome scalability and deployment cost issues of CDNs.HB Assisted CDN: i). An efficient and adaptive video content caching that brings video contents more closer to the End-Users permitting thus short end-to-end distances for more controlled risk of service failures.ii).Infinitely scale services by relying on the HB storage and uplink bandwidth capabilities.iii)Dynamically managing the user requests.iv).HB can act as a Content/Service Provider for the contents published by its related users.v).HBs are organized in an overlay and act both as proxies for their "home serving" users when they request a service and as a server for supplying other HBs related clients with the video contents stored in their caches.Popularity Based Video Caching Strategy: HB maintain the particular video replication strategy. It make available the video contents to the possible users' demand, reducing delays and network bandwidth consumption.Each participating HB contributes with persistent disk storage capacity and upload bandwidth. All HBs have the same storage capacity and upload connectivity. Important to replicate videos in the overlay according to their popularity. How to better distribute them among the HBs? An efficient distribution, we have used the K- means algorithm to determine the distance to respect between any two HBs that will cache the same video content. 


\section{H. On Incentiving Caching For P2P-VOD Systems(RBS)}

Incentive scheme that peers to cache the needed video data. A reward-based incentive scheme for caching, where the content provider decides the reward price of each video and peers decide what videos to cache in a distributed manner. We apply a mean field model to characterize the steadystate of the caches in a large scale $\mathrm{P} 2 \mathrm{P}-\mathrm{VoD}$ system. We formulate a pricing problem using an optimization framework and solve the optimal prices that minimize the content provider's operational cost. Our contributions are: 1).We develops a stochastic model to characterize the peers' caching behaviors, and use the mean field technique to characterize the system state in a limiting steady state.2).We formulates an optimal pricing problem of the content provider and derives the optimal reward prices for each video. Our scheme incentivizes the peers to cache enough replicas of various videos in the system and minimizes the content provider's operational cost.[9]. In Existing approach A straight-forward way is using a stochastic model where the system's state is determined by all peers' cache states, such a system can be computationally expensive. When the number of peers is large, the state space of the system becomes huge. In our incentive scheme to overcome this difficulty, we model the peers in the system using a mean field approach, where we are more interested in the fractions of peers in certain states in steady state, rather than the exact number of peers in the system.Pricing Scheme: The content provider proposes the incentive scheme in order to reduce its operational cost. We define two kinds of pricing strategies.1).Conservative pricing problem (CPP): The content provider would like to set the prices such that the number of cached replicas can satisfy all peers' demand.2).Strategic pricing problem(SPP): when increasing operational cost and upload cost of cache storage, content provider to set lower prices so as to reduce the reward cost and balance the overall utility.

\section{Two-Level Result Caching for Web Search Queries on Structured P2P Networks(TLC)}

Each peer maintains a LRU result cache (RCache) used to keep the answers for queries. To achieve a short-term fair distribution of queries we introduce in each peer a location cache (LCache), which keeps pointers to peers that have already requested the same queries in the very recent past. These peers share their query answers with newly requesting peers. [8] This process is fast as these popular queries are usually cached in the first DHT hop. Then requesting peer which quickly tends to redistribute load among more and more peers. It achieves better load balance, Significantly smaller communication volume among peers, Larger cache hit ratio.DHT (Distributed Hash Table): It provides high data availability and persistence through replication. Every peer associated with unique identifier, which defines the peer's position in the structure, and the range of keys it is responsible for. DHT overlays maintain a strong topology. Every peer assigned a unique nodeID in a space of 128-bits identifiers generated using a cryptographic hash SHA-1. Leafset: that contains the L numerically closest peers. It handles replicas to improve fault tolerance in an environment, where peers join and leave the network without warning. In order to maintain these two sets of peers, a "keep alive" message is used periodically to detect peer failures. Bubble : grows in relation intensity of flood, until no peer in the boundary. It observes high.RCache : The RCache is used to store answers to queries in a normalized format. This cache stores answers of queries for which the peer is responsible, and answers of queries delivered to users that submitted queries in the peer. Each cache entry has a state flag indicating whether the respective query answer is being retrieved from some other place such as a peer or a Web search engine. A timeout flag indicating the time instant from which the respective query answer is no longer valid. RCache entries are administered with the LRU 
replacement policy.LCache: The LCache stores data used to support short-term scheduling. Each entry stores terms of a query, an IP address of a peer that contains (or is to contain soon) the answer for the query, and an expiration counter used to limit the number of times the entry is used to route incoming queries with the same terms. These cache entries are also administered with the LRU replacement policy though their overall size is much smaller than in the RCache as they do not store query answers.

\section{J. Proportional Replication in Peer-to-Peer Networks(PRA)}

In $p 2 p$ networks, that has number of replicas of each object proportional to the request rate for this object and analyzes the how many nodes per advantages. It achieves proportional replication in decentralized system. It using LRU (Least Recently Used) algorithm achieves near proportional replication and replica distribution achieved LRU very close to optimal. If the objective is to minimize the network bandwidth used, the download source should be the nearest replica if multiple replicas of the file are available.Link Distance to the Nearest Replica: To replicate object based on the distance. A client request object to another client. The receiver of client in far away distance, so network bandwidth utilization in increased. The replicate objects on near optimal to reduce bandwidth consumption.Cache Replacement Algorithms for Proportional Replica Distribution: A peer-to-peer system where the peers had finite storage space and if space was needed for a newly requested file then a previously obtained file was deleted using the LRU file replacement policy (except that the last replica of a file is never deleted). The steady-state distribution of the number of replicas of each file against the file request rate. The LRU cache replacement policy obtains near linear proportionality except for high request rate files. Cache management algorithms such as FIFO (First-In, First-Out: replace the oldest file), LFU (Least Frequently Used: replace the least frequently used file) and Random-Delete (randomly select the file to be replaced). All these algorithms generate a replica distribution similar to LRU: LFU is closer to the optimal distribution than LRU while FIFO and RandomDelete are slightly further from it than LRU. LRU: cache replacement algorithms such as LRU are able to achieve near-proportional distribution. Since the user access patterns may change over time. The time taken by LRU to converge to the new steady-state replica distribution after a change in the user access pattern was found to be very close to the minimum required by any cache replacement algorithm. LRU cache replacement algorithm is a very attractive in network system.

\section{K. RESTREAM - A Replication Algorithm for Reliable and Scalable Multimedia Streaming}

In this paper, an adaptive replication algorithm that relies on replication to achieve reliable and scalable streaming in resource constrained system. It dynamically adapts replica replacement to maximize the number of consumer and reduce latency. Restream supports partitioning method. It performs 1). Serving Consumers 2). Adapting Replication Scheme. In serving consumer, when new consumer enter in the system replication joining on that consumer and leaving in other one. It informs that out of our replication. Adapting Replication, it moves from one state to another state, replication will be growing or shrinking .[10]Node or Link Failure: If node failure case, the failure node eliminate, reconnect with another node. If link failure case, it will not reconnect in structure of current tree immediately. But may be used later. Dynamically Adapting Replication Scheme maximize the number of consumers being concurrent served, without exceeding bandwidth capacity and latency. In partitioning helps to recover the failure and achieve good performance in dynamic changing model. 


\section{QoS-Aware Intelligent Replica Management Architecture for Content Distribution in P2P Overlay Networks (QIRMA)}

In this paper, using Intelligent Replica placement algorithm, which the requested contents are classified into class I and class II. In class I most frequently accessed contents are replicated in strong cluster which is having high weight values and in number copies. In class II least frequently accessed contents are replicated in weak cluster which is having low weight values and in less number of copies.Routing Technique: Routing is performed hierarchically by broadcasting the query only to the strong clusters. This method has a caching technique, to reduce the search latency. Using this method System scalability can be improved by distributing the load across multiple servers which is proposed by replication. The demanded contents can be brought much closer to the clients through replication techniques, thus reducing both the access latency and network traffic and increase the system performance by avoiding hot peers which becomes a bottleneck. Limitation: The limitation in this method is that in strong clusters a few replicated items have more number of copies. So, this consumes more cost, memory space, bandwidth and redundancy. In weak clusters a few replicated items have less number of copies. Increased user response time and performance are the bottleneck of the system.[12]

\section{Distributed Algorithm for the Replica Placement Problem (DARPP)}

Caching and Replication of most popular data objects results in reduction of network bandwidth usage and the access latency by designing a distributed approximation algorithm. The main focus is to improve the efficiency of popular object replication within a distributed replication group.[15] the group may consist of several servers that allocate certain amount of memory to place the replicated object which is requested by their clients. For example: When a server receives a request from its client, it response if the object is in its local storage otherwise it fetches from other replication servers within the group or from the origin server. By analyzing the request rates for objects and the server capacities the replicas are placed by considering the availability of server capacities.Performance: Replication of popular data objects at a server closer to the user reduces the access time and network bandwidth. Thus it increases the overall system performance with all objects. By comparing with centralized algorithm this shows only one percentage degradation of system performance and not decided how many copies of objects should be replicated in the group.

\section{N. A Framework for Lazy Replication in P2P VoD}

Lazy replication is postpones replication, trying to make efficient use of bandwidth. Lazy replication can decrease server load. Two predicators are plugged to create a lazy replication algorithm. This replication is more efficient than eager replication. It reduces departure misses and exist misses if chunks are replicated to a peer with free cache space. Lazy Replication: It uses of two sub algorithms. 1). A peer departure predictor 2). A chunk request predictor. The peer departure predictor predicts if a peer will depart in the next time interval. The chunk request predictor predicts the future popularity of a chunk. The replication algorithm decides which chunks to replicate, to which peers, and when, given uncertainty about the later arrival request. It is impossible to know exactly when peers will depart and what chunks will be requested. The predictors approximate this knowledge. Lazy replication uses a peer departure predictor to choose when to replicate (just before a peer leaves) and to whom (peers that are predicted to be most unlikely to leave) and uses a chunk request predictor to choose what to replicate (the chunks that 
will be the most popular in the coming sessions). [16]Lazy replication uses the same greedy approach. Replicate the chunk with the highest popularity to the peer with the lowest probability of departing. The two differ in when a chunk is replicated. Lazy replication uses the peer departure predictor to decide both when to replicate and to where. Eager replication is more aggressive than lazy replication because it immediately replicates whenever a peer fetches a chunk.

\section{O. An Efficient Caching Scheme and Consistency Maintenance in Hybrid P2P System(ACMA \& TC)}

P2P networks can be divided into two categories: 1). structured peer-to-peer networks in which peers are connected by a regular topology, and 2). unstructured peer-to-peer networks in which the topology is arbitrary. The objective is to design a hybrid peer-to-peer system for distributed data sharing. Consistency maintenance is propagating the updates from a primary file to its replica. Adaptive consistency maintenance algorithm (ACMA) maintains that periodically polls the file owner to update the file due to minimum number of replicas consistency overhead is very low. Top Caching (TC) algorithm helps to boost the system performance and to build a fully distributed cache for most popular information. Caching scheme can reduce query delay, better load balance and higher cache hit ratios. It utilizes both the efficiency of the structured peer-topeer network and the flexibility of the unstructured peer-to-peer network, and good balance between the efficiency and flexibility.[13] To maintain consistency, using file consistency algorithm for hybrid $\mathrm{P} 2 \mathrm{P}$ system so that periodically the file owner to update the file due to number of replicas consistency overhead is very low. It boosts the performance of hybrid P2P, Top Caching (TCS) algorithm is used to build a fully distributed cache for popular information in P2P systems. It effectively relieves the over- caching problems for the most popular objects.

Table : Comparison of Various Caching and Replication Algorithms

\begin{tabular}{|c|c|c|c|c|}
\hline $\begin{array}{c}\text { Algorit } \\
\text { hm } \\
\text { (or) } \\
\text { Techni } \\
\text { ques }\end{array}$ & Advantage & Result & $\begin{array}{c}\text { Improved } \\
\text { Parameters }\end{array}$ & Limitation \\
\hline $\begin{array}{c}\text { WRCR } \\
\text { [3] }\end{array}$ & $\begin{array}{c}\text { It reduces cache } \\
\text { space }\end{array}$ & $\begin{array}{c}\text { Min of } 31 \text { object } \\
\& \text { max of } 177 \\
\text { object }\end{array}$ & Network bandwidth & Delay \\
\hline $\begin{array}{c}\text { MDC } \\
\& \\
\text { MRU[1 } \\
]\end{array}$ & $\begin{array}{l}\text { Reduce package } \\
\text { losing problem } \\
\& \text { server load }\end{array}$ & $\begin{array}{c}\text { Maintain } \\
\text { leading object } \\
\text { only }\end{array}$ & $\begin{array}{l}\text { Uses for high churn } \\
\text { rate }\end{array}$ & $\begin{array}{c}\text { No. of Video } \\
\text { watching rate is } \\
\text { limited }\end{array}$ \\
\hline $\begin{array}{l}\text { FSS \& } \\
\text { CBS[2] }\end{array}$ & $\begin{array}{l}\text { Reduces Client } \\
\text { waiting time, } \\
\text { and consistency }\end{array}$ & $\begin{array}{c}\text { FSS more } \\
\text { consistency than } \\
\text { CBS } \\
\end{array}$ & $\begin{array}{c}\text { Availability of } \\
\text { object }\end{array}$ & $\begin{array}{l}\text { Missing contents on } \\
\text { server }\end{array}$ \\
\hline $\begin{array}{c}\text { PALC }[ \\
5]\end{array}$ & $\begin{array}{l}\text { Achieves high } \\
\text { hit ratio, increase } \\
\text { cache contents }\end{array}$ & $\begin{array}{c}\text { Achieve } 95 \% \text { of } \\
\text { efficiency, } \\
\text { cache hit ratio } \\
90 \%\end{array}$ & $\begin{array}{c}\text { Cache capacity will } \\
\text { improve }\end{array}$ & $\begin{array}{l}\text { Only maintain } \\
\text { popular videos }\end{array}$ \\
\hline
\end{tabular}


International Journal of Computer Science, Engineering and Information Technology (IJCSEIT), Vol.3,No.5,October 2013

\begin{tabular}{|c|c|c|c|c|}
\hline $\begin{array}{l}\text { ARLB[ } \\
4]\end{array}$ & $\begin{array}{l}\text { It adapt all the } \\
\text { video object in } \\
\text { sufficient } \\
\text { bandwidth } \\
\end{array}$ & $\begin{array}{c}\text { Adapt } \\
\text { Homogeneous } \\
\text {,heterogeneous } \\
\text { Peers } \\
\end{array}$ & \begin{tabular}{l}
\multicolumn{1}{c}{ dynamic } \\
changing movie \\
popularity
\end{tabular} & $\begin{array}{l}\text { Computational load } \\
\text { will be increased }\end{array}$ \\
\hline $\begin{array}{c}\text { ORA[7 } \\
]\end{array}$ & $\begin{array}{l}\text { Accurate to find } \\
\text { popular objects }\end{array}$ & $\begin{array}{c}\text { Pop=81\%, } \\
\text { unpop }=10 \%\end{array}$ & Improved optimality & $\begin{array}{c}\text { Suboptimal in } \\
\text { software based }\end{array}$ \\
\hline $\mathrm{HB}[6]$ & $\begin{array}{c}\text { Calculate } \\
\text { distance of end- } \\
\text { user to replicate } \\
\text { same video }\end{array}$ & $\begin{array}{c}\text { Saves } 60 \% \text { of } \\
\text { server load }\end{array}$ & Time complexity & Delay \\
\hline RBS[9] & $\begin{array}{l}\text { High cost } \\
\text { reduction }\end{array}$ & $\begin{array}{c}\mathrm{Cu}=0.2,1,2 \\
2=\text { high price }\end{array}$ & Access Latency & $\begin{array}{l}\text { Dynamically adjust } \\
\text { price for changing } \\
\text { movie popularity }\end{array}$ \\
\hline TLC[8] & $\begin{array}{l}\text { Better load } \\
\text { balance, } \\
\text { High hit ratio }\end{array}$ & $\begin{array}{l}\text { Replication } \\
\text { reduced } 96 \%, \\
10 \% \text { of } \\
\text { improved cache } \\
\text { hit compare } \\
\text { other }\end{array}$ & Waiting time & Delay is increased \\
\hline $\begin{array}{l}\text { PRA[1 } \\
0]\end{array}$ & $\begin{array}{c}\text { It replicate } \\
\text { object based on } \\
\text { user access } \\
\text { pattern }\end{array}$ & $\begin{array}{c}\text { Iteration } \\
\text { increased to } 20 \\
\text { to } 50, \text { maintain } \\
\text { steady state }\end{array}$ & Network bandwidth & $\begin{array}{l}\text { Can't adapt } \\
\text { dynamic user } \\
\text { request }\end{array}$ \\
\hline $\begin{array}{l}\text { RESTR } \\
\text { EAM[1 } \\
2]\end{array}$ & $\begin{array}{c}\text { Good } \\
\text { performance and } \\
\text { recover link and } \\
\text { node failure }\end{array}$ & $\begin{array}{c}\text { Probability by } \\
\text { sending req } \\
\text { leave message } \\
0.5\end{array}$ & Improve access rate & $\begin{array}{l}\text { Bandwidth } \\
\text { consumption }\end{array}$ \\
\hline $\begin{array}{c}\text { LAZY } \\
\text { Replica } \\
{[16]} \\
\end{array}$ & $\begin{array}{c}\text { Decrease server } \\
\text { load }\end{array}$ & $\begin{array}{l}\text { Decrease server } \\
\text { load at } 15 \%\end{array}$ & $\begin{array}{l}\text { Throughput and } \\
\text { latency }\end{array}$ & Cost \\
\hline $\begin{array}{l}\text { ACMA } \\
\& \\
\text { TC[13] }\end{array}$ & $\begin{array}{c}\text { Reduce delay, } \\
\text { better load } \\
\text { balance, high hit } \\
\text { ratio } \\
\end{array}$ & $\begin{array}{c}\text { Decrease join } \\
\text { latency } 2.5 \text { only } \\
\text { other LFR } 7.0 \text {, } \\
\text { LRU } 7.8 \\
\end{array}$ & Speed & Network traffic \\
\hline $\begin{array}{l}\text { DARP } \\
\mathrm{P}[15]\end{array}$ & $\begin{array}{c}\text { Minimizes } \\
\text { Response Time, } \\
\text { Cost \& Increase } \\
\text { Availability }\end{array}$ & $\begin{array}{c}\text { Only } 1 \% \\
\text { degradation of } \\
\text { system } \\
\text { performance }\end{array}$ & $\begin{array}{l}\text { Cost, access latency, } \\
\text { Network bandwidth }\end{array}$ & $\begin{array}{c}\text { Not provide } \\
\text { individual system } \\
\text { performance, } \& \\
\text { number of copies }\end{array}$ \\
\hline $\begin{array}{l}\text { QIRM } \\
\text { A[14] }\end{array}$ & $\begin{array}{c}\text { Access latency, } \\
\text { Network traffic, } \\
\text { Scalability, Fault } \\
\text { Tolerance, } \\
\text { Search Latency }\end{array}$ & $\begin{array}{c}\text { bandwidth } \\
\text { utilization of } \\
\text { QIRM is nearly } \\
80-90 \% \text {, when } \\
\text { compared to } \\
\text { VIRAT, which } \\
\text { is } 60-70 \% \text {. }\end{array}$ & $\begin{array}{c}\text { Throughput, Delay, } \\
\text { Bandwidth, Query } \\
\text { Efficiency, }\end{array}$ & $\begin{array}{l}\text { High availability, } \\
\text { Redundancy, Low } \\
\text { Availability, } \\
\text { Bottleneck } \\
\text { Problem, }\end{array}$ \\
\hline
\end{tabular}




\section{COMPARISON OF PERFORMANCE EVALUATION}

\section{Popularity Aware Limited Caching(PALC)}

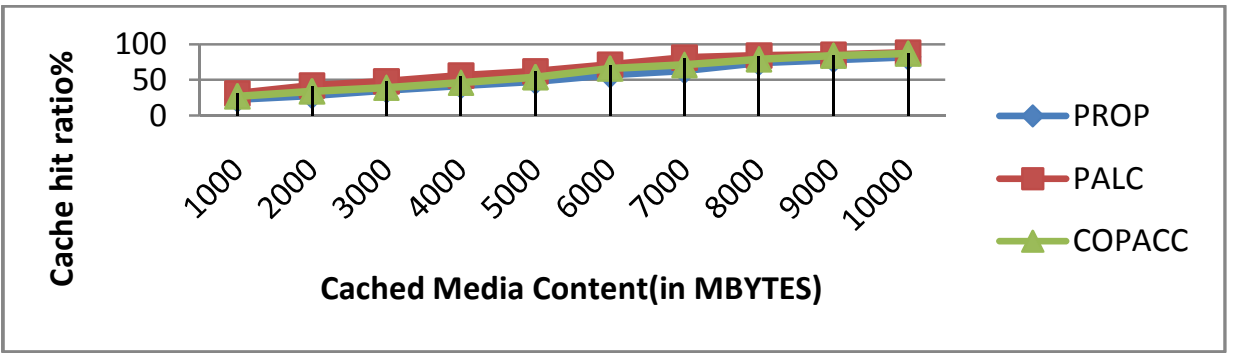

Figure: 1 Cache hit ratio(PALC)

Popularity Aware Limited Caching Obtained upto 90\% of Cache hit Ratio

\section{Two Level Result Caching:(TLC)}

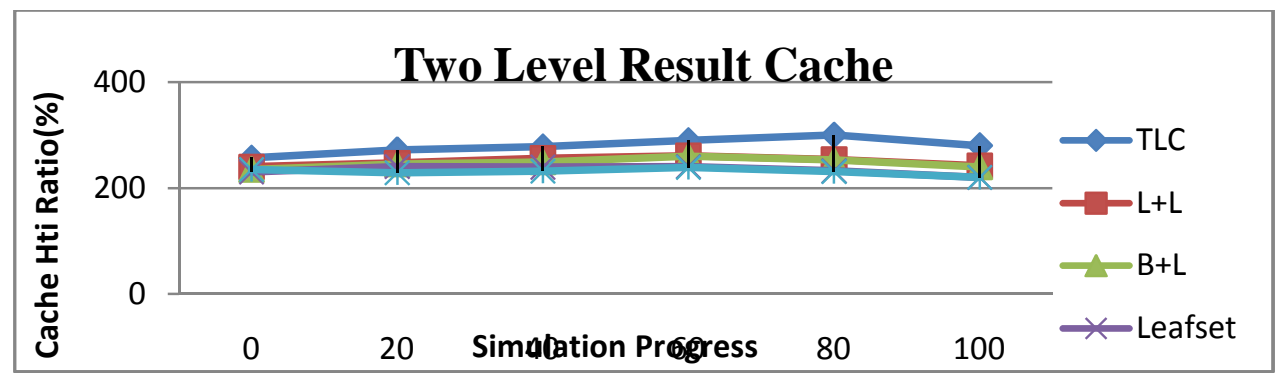

Figure2: Cache hit ratio(TLC)

In TLC improved Cache hit ratio 10\% extra increased for previous one

\section{Home Box Assisted Content Distribution Networks}

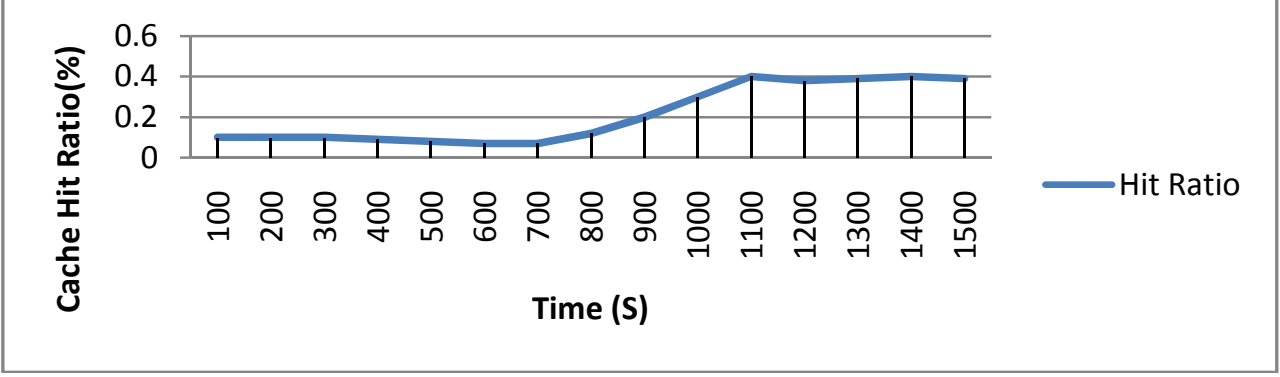

Figure3: Cache hit ratio (HB)

Home Box Assisted Content Distribution Networks Increase Cache hit at 4\% 
In above all caching hit ratio improved is better for Popularity Aware Limited Caching (PALC). It improved up to $90 \%$ cache hit ratio.

\section{Replication Results}

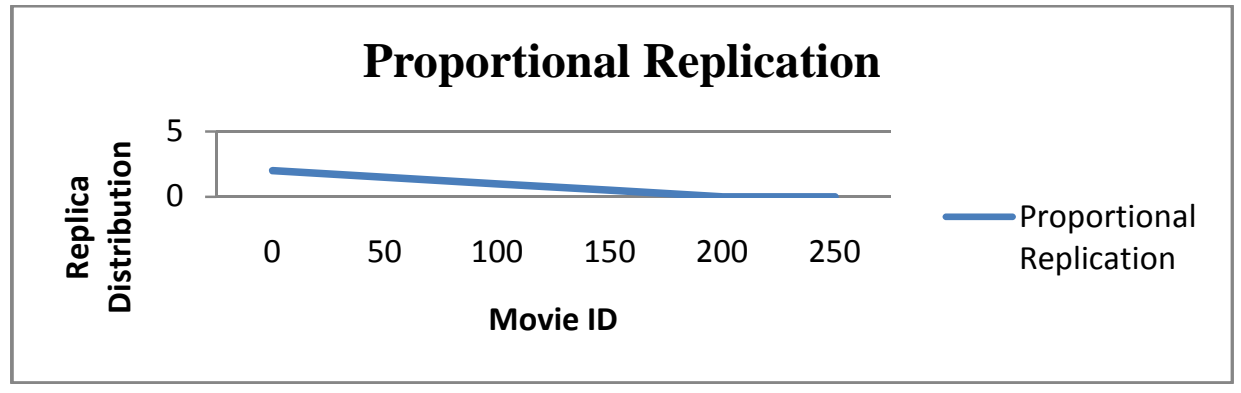

Figure4: proportional Replication

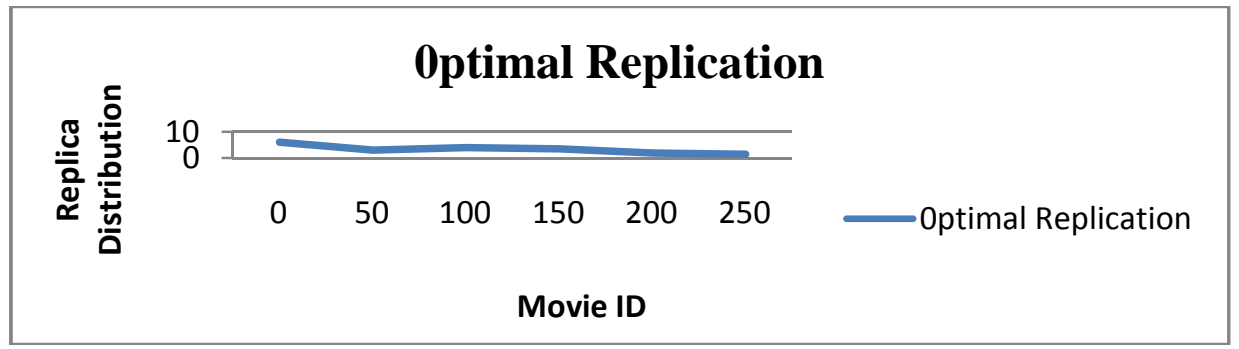

Figure5: Optimal Replication

In proportional and Optimal Replication algorithm, optimal replication distributes more popular videos to consumers. So optimal replication is better than Proportional replication.

\section{Average of Added Additional Objects: WRCR Vs DCRP}

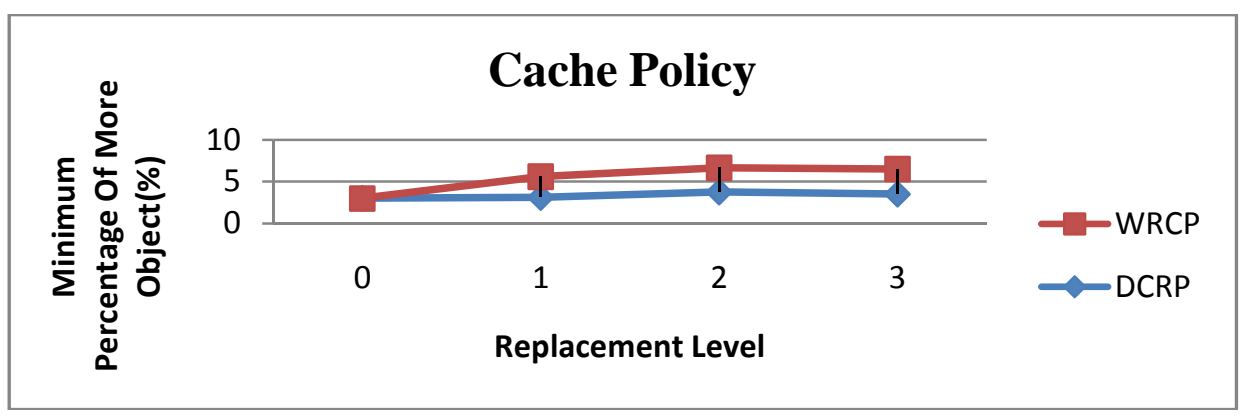

Figure6: Minimum percentage of more object allowed 


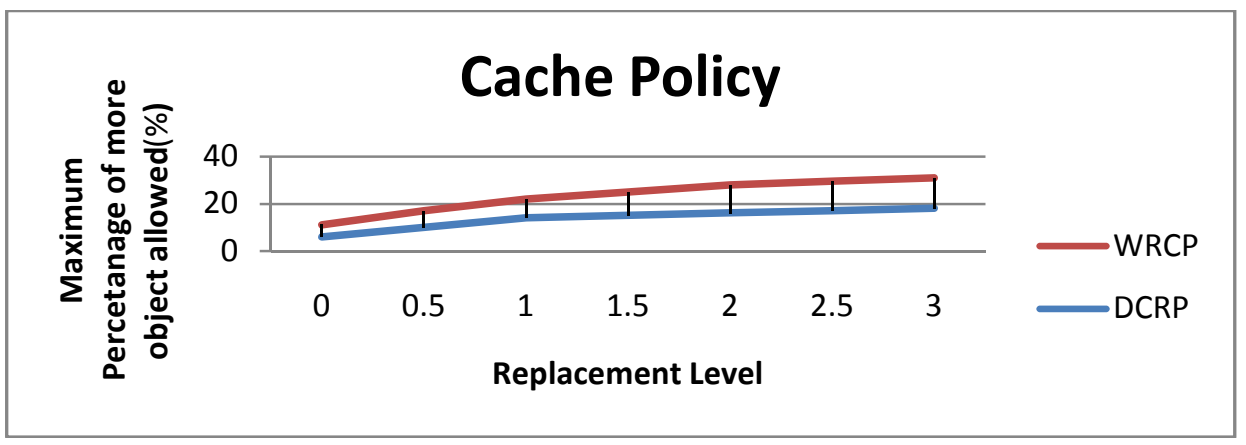

Figure7: Maximum percentage of more object allowed

Compared with DCRP and WRCP, WRCP is the added additional objects. Minimum percentage of adding 31 objects and maximum percentage of adding 177 objects.

\section{Average Bandwidth Utilization: (Home-Box)}

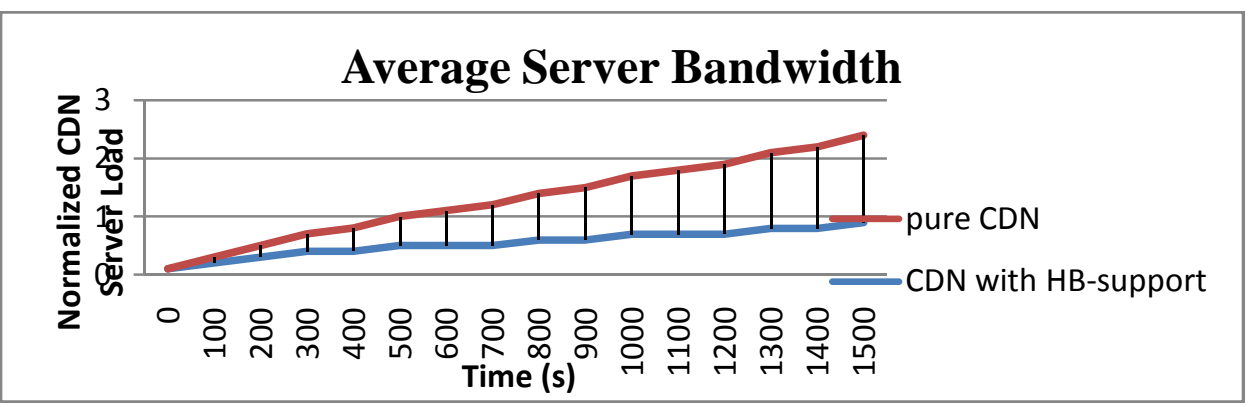

Figure8: HB Bandwidth Utilization

Home box assisted content distribution networks, compared CDN with HB-support decrease server load(10\%) than Pure CDN server load(50\%)

\section{On Replication Algorithm}

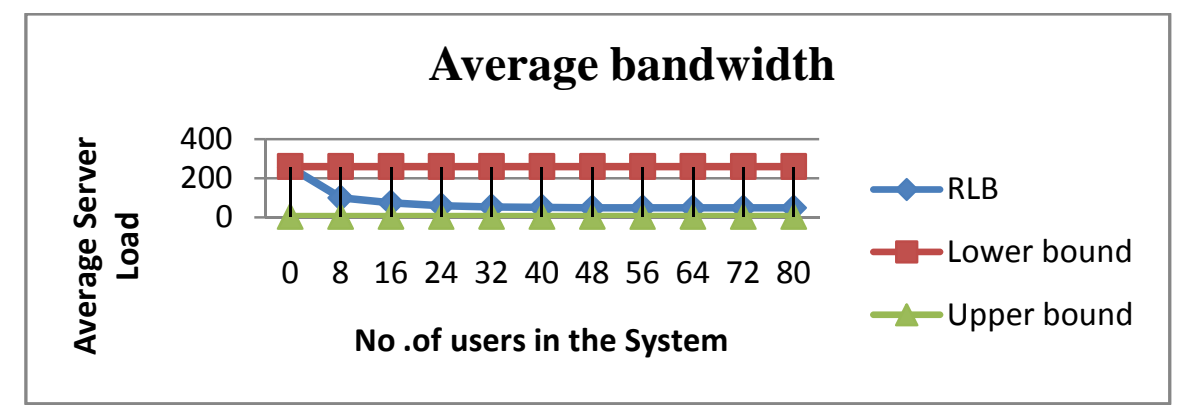

Figure9: Server Load

RLB(Random Load Balancing) balances the server load(50\%), to use sufficient bandwidth for objects replication. 


\section{ACMA and TC: Join Latency Comparison}

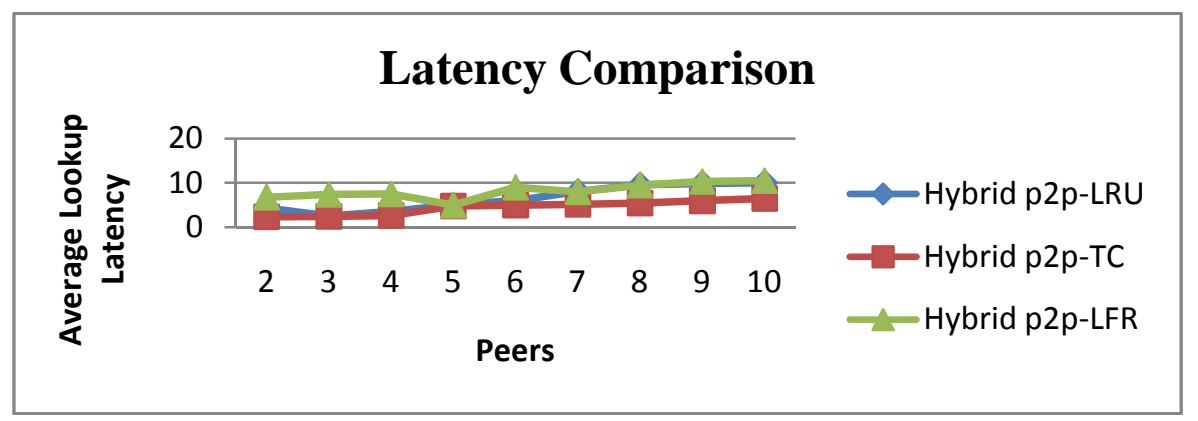

Figure10: Latency

Compared with Hybrid LRU, TC, LFR, TC \& LFR increase latency, LRU lower query delay, better load balance.

\section{CONCLUSIONS}

This paper conducts a theoretical analysis study on caching and replication strategies in peer to peer network. A brief discussion of those techniques is summarized. The advantages and limitations of caching and replication techniques are summarized with reference to various issues related to caching and replication techniques in content distribution of peer to peer networks.

\section{REFERENCES}

[1] Chung- yan chen and yu-wei chen, "Design And Analysis of Streaming for P2P VOD with MDC", Business and Information, Vol.3, No.1. (2013)

[2] R.Thiruselvan,"Design of Distributed Prefetching Protocol in Push to Peer Video on Demand System", International Journal of advanced Computer Technology, Vol.2, No.6. (2013)

[3] S.P.Ponnusamy and E.Karthikeyan, "Optimization Cache on Hot-Point Proxy Caching Using Weighted- Rank Cache Replacement Policy”,ETRI Journel, Vol.35, No.4. (2013)

[4] Yipeng Zhou, Tom Z.J.Fu, and Dah Minh Chiu, "On Replication Algorithm in P2P VoD", IEEE/ACM Transaction on Networking, Vol.21, No.1. (2013)

[5] T.Suresh and K. venkatachalapathy, "Popularity Aware Limited Caching for Reliable On Demand P2P Video Streaming",International Journal of Computer Application, Vol.58, No.13. (2012)

[6] Soraya Ait Chellouche, Daniel Negru, Yiping Chen,"Home Box-assisted Content Distribution Network for Internet Video On Demand Services", ISCC/ ICT-ALICANTE. (2012)

[7] Weijie Wu, John C.S, Lui, "Exploring the Optimal Replication Strategy in P2P-VoD Systems: Characterization and Evaluation", IEEE/ACM Transaction on Parallel and Distributed System, Vol.23, No.8. (2012)

[8] Erika Rosas, Nicolas Hidalgo and Mauricio Marin, "Two Level Results Caching for Web Search Quaries On Structured P2P Networks", IEEE International Conference on Parallel and Distributed System. (2012)

[9] Weijie Wu, John C.S. Lui, Richard T.B, “On Incentiving Caching for P2P VoD Systems", IEEE-Part of INFOCOM, 978-1-4673-1017-8/12.,(2012)

[10] Saurabh Tewari and Leonard Kleinrock "Proportional Replication in Peer to Peer Networks", Journal Of High Performance Computing And Networking. ,(2012) 
International Journal of Computer Science, Engineering and Information Technology (IJCSEIT), Vol.3,No.5,October 2013

[11] Nikhil Chaturvedi and Dinesh Chandra Jain "Analysis of Replication and Replication Algorithms in Distributed System", International Journal of Advanced Research in Computer Science and Software Engineering. Vol. 2, No.5. (2012)

[12] Shabnam Ataee, Benoit Garbinato, Fernando Pedonet ,"RESTREAM- A Replication Algorithm for Reliable and Scalable Multimedia Streaming", Parallel and Distribution System. (2013)

[13] E.Kalaivani, J. Selva Kumar, "An Efficient Caching Scheme and Consistency Maintenance in Hybrid P2P System", International Journal of Computer Networks and Wireless Communication, Vol.2. No.1. (2012)

[14] S. Ayyasamy and S.N. Sivanandam, “ A QOS-Aware Intelligent Replica Management Architecture for Content Distribution in Peer-to-Peer Overlay Networks “, International Journal on Computer Science and Engineering, Vol.1, No.2. (2009)

[15] Sharrukh Zaman and Daniel Grosu, "A Distributed Algorithm for the Replica Placement Problem", IEEE Transaction on Parallel and Distributed Systems. Vol. 22, No.9. (2011)

[16] Bin Cheng, Lex Stein, Hai Jin, “A Framework for Lazy Replication in P2P VoD”, ACM 978-1-60588$157-6 / 05(2008)$

[17] Dr.Anna Saro Vijendran1 and S.Thavamani, "Analysis Study on Caching and replica placement Algorithm for Content Distribution in Distributed Computing Networks “, International Journal of peer to peer network, Vol.3, No. 6. (2012) 